\title{
ROYAL ACADEMY OF \\ MEDICINE IN IRELAND
}

\section{IRISH JOURNAL OF MEDICAL SCIENCE}

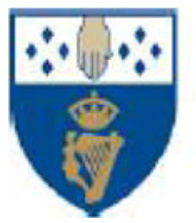

FACULTY OF

Public HEALTh MEDICINE

ROYAL COLLEGE OF PHYSICIANS OF IRELAND

\section{Abstracts}

Summer Scientific Meeting

May 20-21, 2015

\author{
Corrigan Hall, \\ Royal College of Physicians of Ireland \\ 6 Kildare Street \\ Dublin 2 \\ Ireland
}

Irish Journal of Medical Science

Volume 185 Supplement 12

DOI 10.1007/s11845-016-1509-4

黛 Springer 
This supplement was not sponsored by outside commercial interests. It was funded entirely by the Faculty of Public Health Medicine. 


\section{CLICK TO SHARE: SOCIAL MEDIA USE AND RISK OF SEXUALLY TRANSMITTED INFECTION AMONG ATTENDEES OF A SEXUAL HEALTH SERVICE IN MID-WESTERN IRELAND}

\author{
K. I. Quintyne ${ }^{1}$, H. Cowley ${ }^{1}$, J. Clancy ${ }^{2}$, B. Mooka ${ }^{2}$, M. Mannix ${ }^{1}$, \\ A. Dee ${ }^{1}$ \\ ${ }^{1}$ Dept of Public Health, HSE Mid-West, Limerick; ${ }^{2}$ Dept of Infectious \\ Diseases, University Hospital Limerick, Limerick
}

The use of social media (SM) for sexual networking has become increasingly popular within the last 5 years. The current study aimed to determine if SM use for sexual networking among attendees of a sexual health service in UHL was associated with increased incidence of sexually transmitted infections (STIs).

Data were collected from 01/01/2014 to 31/03/2014. A total of 383 attendees at UHL who had undergone STI screening agreed to participate and provided demographic, clinical, sexual health and SM information. These were correlated with serlogical tests in all cases. Regression analysis was performed.

Participants who used SM for sexual networking were more likely to: be male (RR 1.963; 95\% CI 1.612-2.390; $\mathrm{p}<0.001$ ), MSMs (RR 5.834; 95\% CI 3.302-10.300; p, 0.001), $<6$ sexual partners in last 3 months (RR 6.894; 95\% CI 1.984-23.956; $\mathrm{p}=0.002$ ), use alcohol (RR 1.644; 95\% CI 1.315-2.056; $\mathrm{p}<0.001$ ), use recreational drugs (RR 3.586; 95\% CI 1.228-10.471; p < 0.001) and, have an STI confirmed (RR 1.350; 95\% CI 0.981-1.850; $\mathrm{p}=0.066$ ). There were no significant differences in types of STI between those who used SM for sexual networking and those who did not.

The present study concludes that attendees of the sexual health service who used SM for sexual networking were at greater risk of a STI, than those who did not. Future interventions could explore the use of SM for testing promotion, prevention and education on sexual health issues.

\section{SYSTEMIC ANTI-CANCER THERAPY IN IRELAND: A NATIONAL QUALITY IMPROVEMENT INITIATIVE}

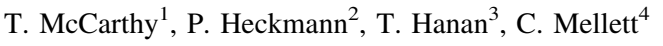 \\ National Cancer Control Programme (NCCP), Dublin
}

Systemic anti-cancer treatment, often referred to simply as chemotherapy, is delivered to approximately 33,000 people in Ireland each year. In 2013, the National Cancer Control Programme (NCCP) commenced a process of assessing and ensuring the safety of treatment delivery.

A review of services, policies and practices in all publicly funded oncology/haemato-oncology day units was conducted under the guidance of a multi-disciplinary steering group and co-sponsored by the HSE Quality and Patient Safety Directorate. The methods used included the completion of a self-administered questionnaire by the 26 hospitals involved and a site visit by a multidisciplinary NCCP review team. Data was analysed using SPSS.

The findings of the review [1] informed the 93 recommendations made by the steering group, in relation to the safe delivery of systemic anti-cancer treatment. The recommendations covered the full scope of treatment planning and delivery in the day unit setting and were cross referenced to the National Standards for Safer Better Healthcare [2].

Some review recommendations were for immediate implementation by hospitals; others related to gaps in national guidance and required the establishment of national working groups. An audit process was established to monitor the implementation of the recommendations (see http://www.hse.ie/nccponcsafetyreview). At the end of 2014, two-thirds of recommendations had been implemented by hospitals and work was underway in relation to $85 \%$ of recommendations requiring the development of national guidance.

\section{THE BURDEN OF UNCONTROLLED DIABETES ON THE ACUTE HOSPITAL SERVICES IN IRELAND 2008-2013. AN IMPORTANT PREVENTION QUALITY INDICATOR}

\author{
A. O’Farrell ${ }^{1}$, R. Canavan ${ }^{2}$, K. Balanda ${ }^{3}$ \\ ${ }^{1}$ Health Intelligence Unit, Health and Wellbeing, HSE; \\ ${ }^{2}$ Dept of Endocrinology, St. Vincent's Hospital; \\ ${ }^{3}$ Institute of Public Health in Ireland
}

This study examines the impact of poorly controlled diabetes on emergency hospital admissions among the estimated adult diabetic population in Ireland.

Emergency in-patient admissions with a principal diagnosis of poorly controlled diabetes (i.e., diagnoses for hyperosmolarity, acidosis, hypoglycemia and poor control) were obtained from Hospital In-Patient Enquiry (HIPE) database for adults (aged 18+ years) from 2008 to 2013. All statistical analyses were carried out in SPSS and StatsDirect. Estimated diabetic population figures were obtained from the Institute of Public Health.

There were 12,695 emergency hospital admissions for uncontrolled diabetes among those aged $\geq 18$ years. Although the number of admissions, median LOS, and estimated hospital costs reduced over the study period, the impact and costs remain substantial. 
Table 1 Burden of emergency uncontrolled diabetic admissions on acute hospitals, 2005-2013

\begin{tabular}{llllll}
\hline Year & $\begin{array}{l}\text { No. of } \\
\text { emergency } \\
\text { admissions }\end{array}$ & Median & $\begin{array}{l}\text { (LOS) } \\
\text { Total } \\
\text { bed } \\
\text { days }\end{array}$ & $\begin{array}{l}\text { Estimated } \\
\text { in-hospital } \\
\text { costs } \\
\text { based on } \\
\text { DRGs } \\
\text { Age }\end{array}$ & $\begin{array}{l}\text { Standardised } \\
\text { hospitalisation } \\
\text { rate per 1000 } \\
\text { estimated } \\
\text { diabetic } \\
\text { population }\end{array}$ \\
\hline 2008 & 2240 & 4 & 15373 & $€ 12,696,410$ & 37.7 \\
2009 & 2149 & 4 & 15829 & $€ 13,533,614$ & 37.0 \\
2010 & 2099 & 3 & 13772 & $€ 11,754,841$ & 36.3 \\
2011 & 1958 & 3 & 13461 & $€ 11,127,745$ & 33.6 \\
2012 & 2098 & 3 & 13700 & $€ 10,732,088$ & 34.6 \\
2013 & 2151 & 3 & 12286 & $€ 11,262,916$ & 34.5 \\
$2008-2013$ & 12695 & 3 & 84221 & $€ 71,127,613$ & \\
\hline
\end{tabular}

This study found that despite an increase in the rate of diabetes in the population, the rate of hospitalisations for uncontrolled diabetes has decreased. However, the cost burden from potentially avoidable hospitalistions remains high, particularly in a time of reduced resources. Improved accessibility to ambulatory care is likely to lead to a significant reduction in avoidable emergency admissions.

\section{UTILISATION AND TEMPORAL TRENDS FOR ELECTIVE ORTHOPAEDIC SURGERY IN IRELAND}

\author{
R. Glynn ${ }^{1}$, P. Harrington ${ }^{2}$, M. O’ $\mathrm{Neill}^{2}$, M. Ryan ${ }^{2}$
}

\section{${ }^{1}$ Dept of Public Health, HSE East, Dublin; ${ }^{2}$ Health Information and Quality Authority}

The requirement for standardisation of surgical practice is increasingly relevant as changing demographics and the increasing prevalence of both obesity and chronic disease place additional strain on publicly-funded healthcare systems. This work aimed to establish trends in utilisation of elective orthopaedic services within the publicly-funded healthcare system in Ireland.

The Hospital In-Patient Enquiry (HIPE) system was employed to assess activity levels and temporal trends in relation to the procedures under review, and the budget impact of each on the public healthcare system was established. Overall activity levels, day case rates, and hospital length of stay were analysed.

Between 2005 and 2012, the number of knee and shoulder arthroscopies performed annually increased by 39.2 and $164 \%$, respectively. Increases in day-case rates for both knee and shoulder arthroscopy were noted, as were significant reductions in average length of stay for those having knee and hip arthroplasty. There is persistent variation in length of stay and day case rate at local level however. The current estimated costs of elective hip arthroplasty (annual national cost $€ 37.3$ million, average weighted cost per case of $€ 11,403)$, knee arthroplasty (€25.6 million, $€ 11,925)$, shoulder arthroscopy ( $€ 2.9$ million, $€ 3,701)$ and knee arthroscopy ( $€ 10.3$ million, $€ 2,457)$ were calculated.

Significant efficiencies have been achieved in the provision of elective orthopaedic surgical services in Ireland. Deficiencies remain to be addressed however, with a need in particular for analysis of the factors contributing to variation at local hospital level.

\section{DENTAL ATTENDANCE AND SELF-REPORTED ORAL STATUS AMONG ADULTS AT HIGH RISK FOR ORAL CANCER}

\author{
P. Fitzpatrick ${ }^{1}$, M. McGeown ${ }^{1}$ \\ ${ }^{1}$ University College Dublin
}

Oral cancer prognosis can be greatly improved with early detection. In the absence of a national screening programme individuals need to access dental care regularly to allow for opportunistic screening. Known risk factors for oral cancer include age, gender, smoking and higher alcohol consumption. The aim of this study was to assess the relationship between high risk oral cancer patients, dental attendance and self-reported oral status.

Data was analysed from the SLÁN 2007 dataset of 10,364 adults living in Ireland. Binary and multinomial logistic regression was used to determine the odds ratios for identified risk factors for oral cancer and (1) attendance at a dental professional within the preceding 12 months and (2) self-reported oral status.

Males, those $\geq 45$ years and current smokers were less likely to have attended for a dental check-up within 12 months $(\mathrm{p}<0.05)$ and more likely to report missing teeth $(\mathrm{p}<0.05)$. Current smoking significantly reduced the likelihood of regular dental attendance $(\mathrm{p}<0.05)$, while current/ex-smoking and drinking above weekly recommended limits did not $(\mathrm{p}>0.05)$. Medical card holders were significantly less likely to have attended and more likely to report missing teeth while holders of private health insurance were more likely to attend and reported fewer missing teeth $(\mathrm{p}<0.05)$.

Those with multiple risk factors for oral cancer are less likely to attend for routine dental check-ups to allow for opportunistic screening of oral cancer and are more likely to report missing teeth. All health professionals play an essential role in encouraging routine dental attendance among their patient

\section{OPPORTUNISTIC SCREENING FOR ATRIAL FIBRILLATION THROUGH PRIMARY CARE IN A RURAL AREA}

\author{
B. Smyth ${ }^{1}$, P. Marsden ${ }^{2}$, R. Corcoran ${ }^{1}$, R. Walsh ${ }^{3}$, C. Brennan ${ }^{2}$, \\ K. McSharry ${ }^{2}$, J. Clarke ${ }^{4}$, J. Harbison ${ }^{5}$ \\ ${ }^{1}$ Dept of Public Health, HSE West, Galway; ${ }^{2}$ Dept of Public Health, \\ HSE Midlands, Tullamore; ${ }^{3}$ Galway University Hospital; ${ }^{4} G P$ \\ Clinical Care Programme; ${ }^{5}$ Trinity College, Dublin
}

Atrial Fibrillation (AF) is associated with a fivefold increased risk of stroke and this increases with age (1). There is debate whether opportunistic or organised screening is more effective in identifying cases of AF. Previous screening studies have typically been carried out in highly populated areas (2). In rural areas, organised screening and management of AF is more challenging because of reduced proximity and access to primary and secondary care and difficulty accessing specialist coagulation services. This study investigated the feasibility of AF screening in two predominantly rural counties in the West of Ireland.

A selection of General Practices participated in screening consecutive subjects aged $>64$ years over a 6-month period using pulse measurement, supported with 12-lead electrocardiogram where arrhythmia was suspected. Screening was supported with a management algorithm computerised collection tool.

A total of 7262 individuals were screened. Irregular pulse was identified in $916(12.6 \%)$ patients and 55 patients $(0.8 \%)$ had 
previously undiagnosed AF. Of these new AF, 27 (50\%) were asymptomatic on presentation and all patients had AF confirmed on practice electrocardiogram. A CHA2DS2VASc score was recorded for 39 of the 55 patients $(70.9 \%)$ and 37 of these (94.9\%) had a score $>2$ and were at high enough risk of stroke to be considered for anticoagulation therapy. Thirty-three patients (61\%) were commenced on anticoagulation therapy either in general practice or in the hospital setting. This indicates a positive change in practice.

The findings reinforce the utility and feasibility of opportunistic screening for $\mathrm{AF}$ in rural areas.

\section{ANALYSIS OF EMERGENCY AND URGENT CARE SYSTEM POLICY IN IRELAND, POLICY COHERENCE AND IMPLEMENTATION}

\author{
C. Buckley ${ }^{1}$, O. Healy ${ }^{1}$, E. Droog ${ }^{2}$, S. Foley McHugh ${ }^{2}$, J. Browne ${ }^{2}$ \\ ${ }^{1}$ Dept of Public Health, HSE South, Cork; ${ }^{2}$ University College Cork
}

A large proportion of healthcare resources are being diverted into the Emergency and Urgent Care System (EUCS). This aim of this study was to describe the evolution of policy relevant to EUCS in Ireland, using the Health Policy Triangle, to examine coherence between different policies and to assess policy implementation.

A retrospective documentary analysis of national and regional policy documents related to the EUCS was conducted. A framework approach was used to analyse the documents, identifying context, actors, content and processes.

Policy context is influenced by the socio-political, economic and cultural environment. The Churches and medical profession were dominant actors but recently national and international regulators, the media and the general public are visible. Drivers include quality, access and equity, specialisation, patient safety, clinical governance and cost. Three types of evidence informing policy formation were identified; expert opinion, peer-review research, and assessment of the status quo. The use of evidence has improved over time. Public consultation has also improved over time.

EUCS policy demonstrates both coherence and inconsistency. The recommendations of all policies relate to a new model of care, with centralisation of acute complex care, and the development of critical enablers needed to achieve implementation. However, incoherence emerges in implementation, partially attributable to changing governance and management structures.

Emergency and urgent care is a developing area. There are sustained efforts to develop a systems approach. Public opinion when expressed is often negative as service user involvement particularly in policy and health service development is piecemeal.

\section{DEVELOPMENT OF NATIONAL ELECTRONIC CANCER REFERRAL IN IRELAND: A SOLUTION FOR SAFER REFERRAL PATIENT PATHWAY}

\author{
E. Nolan ${ }^{1}$, M. Laffoy ${ }^{1}$, V. Jordan ${ }^{2}$ \\ ${ }^{I}$ National Cancer Control Programme (NCCP); ${ }^{2}$ ICT Directorate, \\ HSE
}

The National Cancer Strategy (1) recommended that GPs should have comprehensive information available to them, to enable them to assess a patient with suspicious symptoms. Primary care is often the first contact with the health system. The needs of GPs in relation to cancer, were identified in a ICGP report (2).

The development of electronic referral systems aims to increase the number of patients diagnosed at an earlier stage and maximise the potential for cure. This is a multi-agency, and involves the NCCP, Healthlink, ICGP, ICT Directorate, HSE and GPIT. The electronic referral process takes place via The National Healthlink Project (http://www.healthlink.ie).

Phase one involved developing site-specific cancer referral forms in Healthlink. Referral guidelines and paper referral forms (breast, prostate and lung) were developed by the NCCP.

Phase two was the development of breast, prostate, lung and skin cancer electronic cancer referral via the four ICGP accredited GP practice management software systems.

Phase three is the development of an integrated web services browser using the NCCP Pigmented Lesion GP Referral Form. This will facilitate the development of future electronic referral forms.

$46 \%$ of all breast, prostate and lung cancer referrals were sent electronically in quarter one 2014. This project will streamline the cancer referral process and provide rapid access for patients who are being referred with a suspected cancer.

1. A Strategy for Cancer Control 2006, National Cancer Forum, $\mathrm{DOH}$.

2. Early Detection of Cancer, A Needs Assessment of General Practitioners, Irish College of General Practitioners, 2007

\section{OUTBREAK OF LYMPHOGRANULOMA VENEREUM (LGV)}

\section{F. Cooney ${ }^{1}$ \\ ${ }^{1}$ Dept of Public Health, HSE-East, Dublin}

This report describes the first lymphogranuloma venereum (LGV) outbreak notified in Ireland. LGV is a sexually transmitted infection of significant public health importance. It is caused by infection with the L1, L2, and L3 serovar strains of Chlamydia trachomatis.

The outbreak is currently centred in the greater Dublin area and, at the time of writing, there have been 43 cases of LGV notified since May 2014. To date, all cases are among men who have sex with men (MSM), the majority are HIV-positive and median age is 35 years with age range of $20-55$ years. Available data indicate that the epidemiology of this outbreak is similar to that described in other Western countries.

In October 2014 Public Health convened a multidisciplinary LGV outbreak control team (OCT) to investigate and control the outbreak. Control measures include active case finding and contact tracing as well as an information campaign targeted at men at risk, which included the development of a patient information leaflet and poster. The STI/HIV clinics are being requested to play an important and proactive role in raising awareness and offering clinical assessment to MSM at risk of LGV infection.

Molecular virology investigations indicate that most of the cases may be very closely linked. Gene sequencing carried out on the outer membrane protein A (ompA) gene has demonstrated that of the 40 isolates tested, 36 have identical DNA sequences to the L2 Ref/434 strain.

The results from the ongoing epidemiological investigation will inform further control measures. 


\section{MALE PERCEPTIONS AND INVOLVEMENT IN CHILDBIRTH: KEY FOR REDUCING MATERNAL MORTALITY IN RURAL ZAMBIA}

\author{
V. Mukonka ${ }^{1}$, F. McAuliffe ${ }^{2}$, O. Babaniyi ${ }^{3}$, J. Mwanza ${ }^{4}$, \\ P. Fitzpatrick ${ }^{1}$
}

${ }^{1}$ Copperbelt University/University College Dublin, School of Public Health, Physiotherapy \& Population Science, Dublin; ${ }^{2} U C D$ Obstetrics \& Gynaecology, School of Medicine \& Medical Science, Dublin; ${ }^{3}$ World Health Organisation, Country Office, Lusaka; ${ }^{4}$ University of Zambia, School of Humanities \& Social Sciences, Lusaka

Institutional delivery by skilled birth attendants is considered single most important strategy to reduce maternal mortality ratio (MMR) in developing countries (1). MMR in Zambia (591/100,000 live births) is among the highest worldwide. As part of a study looking at barriers to institutional deliveries and a community intervention trial evaluating the effect of provision of non-financial incentives on increasing institutional deliveries in Monze district, Zambia, the aim of this study was to gain understanding of husbands' perceptions of and role in decisions regarding choice of delivery location.

A qualitative exploratory study using in-depth interviews was conducted in Nampeyo, a rural region in Monze. Purposive sampling, using snowball technique and maximum variation sampling was used to select 27 participants. Qualitative data was recorded, transcribed and translated into English. Content analysis framework was employed for analysis.

The final decision regarding place of delivery was made by men in consultation with their wives, sometimes with relatives. While husbands appreciated to some extent the benefits of institutional deliveries by skilled birth attendants, perceived barriers to institutional deliveries were demands made and items needed for facility delivery, disruption of normal home life, unwelcoming health facility environment and negative staff attitude. Perceived roles and responsibilities included provision of items needed for institutional delivery, mobilising resources, transport, undertaking home duties to support their wives during pregnancy and childbirth.

Study results will assist in the design of effective policy for improving institutional deliveries and reduction of MMR that will reflect the cultural importance of positive male engagement.

\section{THE CHANGING EPIDEMIOLOGY OF NEWLY DIAGNOSED HIV INFECTIONS IN CLARE, LIMERICK \& TIPPERARY NORTH, 2010-2014}

\author{
K.I. Quintyne ${ }^{1}$, M. Morris-Downes ${ }^{1}$, R. Fitzgerald ${ }^{1}$, M. Mannix ${ }^{1}$ \\ ${ }^{I}$ Dept of Public Health, HSE Mid-West, Limerick
}

To study current epidemiology of newly diagnosed HIV infections in Clare, Limerick \& Tipperary North (HSE MW) and describe temporal trends

Data were collected from 01/01/2010 to 31/12/2014 from enhanced surveillance records for HSE MW. Ninety-one cases were identified. Descriptive analysis of demographic and clinical information was performed.

In 2014, 18 new cases of HIV were diagnosed, representing a 29\% increase compared with 2010. Between 2010 and 2014, average age of cases declined from 35 to 32 years and male: female ratio remained unchanged. The proportion of cases born outside of Ireland increased from 21 to $44 \%$. This was associated with an increase in cases of Black ethnicity from 7 to $17 \%$. MSM transmission was the most common in 2014, showing an increase from 43 to $67 \%$ over the interval. The median CD4 count remained unchanged. The median viral load increased from 12012 to 21776 copies $/ \mathrm{ml}$. The proportion of cases presenting asymptomatically decreased from $67 \%$ in 2010 to $50 \%$ in 2014. The proportion of cases presenting with co-infections with Syphilis and Hepatitis C both increased (0-17 and 0-6\% respectively), while other STI co-infections patterns remained unchanged.

There has been an increase in the annual number of cases of newly diagnosed HIV. Changes in the epidemiology of newly diagnosed HIV were seen by age, mode of transmission, country of birth and coinfections with STIs. These changes and increases have implications for the prevention, planning and financing of HIV services in HSE MW.

\section{CUTANEOUS DIPHTHERIA IN A HIGHLY VACCINATED POPULATION}

\author{
L. O' Connor ${ }^{1}$, M. Ward ${ }^{1}$, S. Cotter ${ }^{2}$, P. Murphy ${ }^{3}$, H. Murray ${ }^{1}$, \\ N. Leonard ${ }^{4}$
}

${ }^{1}$ Dept of Public Health, HSE East, Dublin; ${ }^{2}$ Health Protection Surveillance Centre, Dublin; ${ }^{3}$ Dept of Microbiology, Adelaide, Meath and National Children's Hospital, Tallaght, Dublin 24; ${ }^{4}$ School of Veterinary Medicine, University College Dublin, Belfield, Dublin 4

Diphtheria is a toxin-mediated disease resulting from infection caused by toxin-producing Corynebacterium diphtheriae, C. ulcerans and C. pseudotuberculosis. Clinically, diphtheria presents as acute upper respiratory tract infection or cutaneous infection. Toxinmediated complications include myocarditis and neuritis. Diphtheria is rarely seen in Ireland due to high vaccination coverage in the population.

In February 2015, a consultant microbiologist notified the Department of Public Health of the isolation of toxin-producing Corynebacterium from a palmar abscess in an Irish adult. A diagnosis of cutaneous diphtheria, due to $C$. ulcerans, was made. Following antibiotic treatment, the case recovered fully. Diphtheria anti-toxin was not given.

Public health actions for eight close contacts included; taking nasal and pharyngeal swabs which were sent to the Diphtheria Reference Laboratory for analysis, antibiotic chemoprophylaxis and appropriate diphtheria vaccination. As literature review and consultation with expert veterinary colleagues supported a domestic animal as the most likely infection source, microbiological samples were collected from three asymptomatic domestic animals with which the patient had frequent contact. These were sent to the School of Veterinary Medicine at University College Dublin for analysis. All human and animal microbiological samples tested negative for diphtheria.

Despite negative microbiological results, a domestic animal infection source was thought to be most likely. C. ulcerans has been isolated increasingly in domestic animals and several recent human cases in the UK have been linked to domestic animals $(1,2)$. While diphtheria is extremely rare, this case highlights the importance of maintaining high vaccination coverage at population level to prevent such infections. 


\section{PROMOTING WATER INSTEAD OF SUGARY DRINKS: A MULTIFACETED APPROACH}

\author{
M. Faughnan \\ ${ }^{1}$ Safefood
}

Strong evidence exists that sugar sweetened beverages (SSB) consumption promotes weight gain in children and adults (1). One in four Irish children aged between two and 12 years is carrying excess weight (2). Children consume $5 \%$ of their total daily dietary energy from $\operatorname{SSB}(3,4)$.

The childhood obesity campaign launched in October 2013 provides parents with practical solutions in tackling childhood obesity. Swapping sugar drinks for water was one of the six key messages of the campaign. The key activities took place to promote the message:

A survey of the sugar content of common SSB on the market on the island of Ireland was conducted and presented as an info graphic that could be disseminated via digital and social media to consumers and health professionals; Television, radio and outdoor advertisement; PR: A poster of the drinks infographic was disseminated to dental practices.

Benchmark research was conducted with 909 parents of 1-12 year olds across the island of Ireland in September 2013 using face to face interviews. The research was repeated in September 2014 with 567 parents. The findings found that the consumption of SSB by children once a day or more dropped from 45 to $40 \%$ and the reported increase in consumption of water at mealtimes rose from 31 to $38 \%$. The sugar content of squashes/cordials and fruit drinks received a lot of media attention. The infographic was found to be an engaging tool by consumers and for health professionals.

\section{A REGIONAL APPROACH TO IMPROVING HEALTHCARE WORKER INFLUENZA VACCINATION UPTAKE-THE DONEGAL STAFF INFLUENZA VACCINATION CAMPAIGN}

\author{
E. Robinson ${ }^{1}$, M. Moran, A. McAteer, L. Thompson, A. Murray, \\ J. Hayes, M. Gordon, Á. Flanagan, L. Doherty ${ }^{1}$
}

\section{${ }^{1}$ Dept of Public Health, HSE Northwest}

Influenza vaccination of healthcare workers (HCWs) protects staff from contracting influenza and thus protects against nosocomial transmission of influenza to patients, service users or other staff. A multitude of agencies and organisations recommend influenza vaccination of HCWs including the WHO and the Council of the EU. This recommendation is endorsed by the National Immunisation Advisory Committee in Ireland. However, HCW uptake of influenza vaccine remains low in Ireland.

In 2013, prompted by staff influenza vaccination uptake rates below national levels and numerous ILI outbreaks in residential settings the then Donegal Community Care Area was eager to increase uptake rates in the region. The Donegal Influenza Vaccination Campaign was established led by a steering group consisting of representatives from Public Health Medicine, Community Infection Prevention and Control, Health Promotion, the acute hospital, HSE Communications, and Management.

Measures implemented to date include recruitment and training of local 'flu champions' from each service area to advocate and educate their peers; a teaser poster campaign featuring local HCWs; a vaccine launch day in 2014 attended by local celebrities. For the 2015/2016 season plans include introducing peer vaccinators and improved monitoring of uptake rates.

The challenges encountered and successes achieved will be discussed.

Improving HCW influenza vaccine uptake rates requires a multifaceted and ongoing approach, thus the Donegal Influenza Vaccination Campaign is an iterative process. Regional campaigns such as this can augment the national approach.

\section{NATIONAL ACUTE MEDICINE PROGRAMME- IMPROVING QUALITY AND PATIENT EXPERIENCE FOR ALL MEDICAL PATIENTS IN IRELAND}

F. Cianci ${ }^{1}$, O. O'Reilly ${ }^{1}$, A. Casey ${ }^{2}$, E. Croke ${ }^{2}$, C. Conroy $^{2}$, A. M. Keown ${ }^{2}$, G. Leane ${ }^{1}$, B. Kearns ${ }^{3}$

${ }^{1}$ Dept of Public Health, HSE South East, Kilkenny; ${ }^{2}$ HSE; ${ }^{3}$ Blackrock Medical Clinic

The National Acute Medicine Programme (NAMP) was established to address the unsatisfactory management of acutely ill medical patients in Ireland. It aimed to improve quality of care and patient safety; streamline access to healthcare and reduce cost through efficiency gains.

A model of care was developed to describe four distinct clinical pathways for acutely ill medical patients streamed from Emergency Departments (ED) to Acute Medical Assessment Units (AMAUs). Specific areas of intervention and the respective practice changes necessary to improve process were identified for each pathway. A patient flow model was used to build system capacity and predict demand for each hospital. A performance framework, with national benchmarks that mirrored the model of care, was also developed. The programme team met regularly with hospitals and fed back performance information and, using appreciative enquiry, supported local improvement plans.

Thirty-two Irish hospitals that admit acute medical patients are now operating the programme and available inpatient data was improved and harnessed to support on-going audit. Between 2010 and 2013, a reduction of 1.6 days in national average length of stay (AvLOS) was achieved. Process improvement lies at the core of this success. However, the national AvLOS has remained static over the last year due to significant egress blockages at the hospital level.

Despite an increase in hospital admissions and severe resource constraints during this time period, the NAMP has achieved significant efficiency gains through process improvements, while ensuring patient safety and improving the quality of healthcare delivered in Ireland

\section{DO IRISH HEALTH SERVICES NEED LEAGUE TABLES?}

\author{
D. Mulholland ${ }^{1}$, G. Cosgrove ${ }^{2}$ \\ ${ }^{1}$ Chief Medical Officer's (CMO's) Office; ${ }^{2}$ Information Unit, Dept \\ of Health
}

As part of a commitment to public reporting of information on quality and safety of healthcare in Ireland, the Minister for Health announced 
the establishment of the National Healthcare Quality Reporting System (NHQRS) in February 2014.

The CMOs office, working with the information unit of the Department of Health, led on this policy. In the last year a governance structure was established and the first annual report of the NHQRS was developed and published.

A multidisciplinary Governance Committee was established to oversee the development of a reporting framework and the selection and analysis of indicators for the first annual report.

The first annual report, published on the 5th March 2015, included information at national level to support international comparison and at regional and hospital level to support comparison between health services in Ireland.

There has been some controversy about the proposed level of reporting of some information for example in-hospital mortality for certain conditions for named hospitals. Therefore the governance structure and the communication plan needed to manage expectations and ensure that the information provided was robust and presented in such a way as not to cause misinterpretation.

The report presented a number of indicators from different sectors of the health services, with commentaries and explanatory notes including any limitations. The information was not presented in league tables.

The information is now in the public domain which is in keeping with the principles of openness, transparency, accountability and empowerment of patients, the public and service providers at all levels.

\section{BULLYING AND HEALTH CARE UTILISATION IN 9-YEAR-OLD CHILDREN: A CROSSECTIONAL STUDY}

Hayes Catherine $^{1}$, U. Reulbach ${ }^{1}$, D. Kelly ${ }^{1}$, E. Nixon ${ }^{1}$, M. O’Moore ${ }^{1}$, J. Williams ${ }^{2}$, T. O'Dowd ${ }^{1}$

${ }^{1}$ Trinity College, Dublin; ${ }^{2}$ ESRI

This study examined the association between self-reported victimisation by bullying in children and health service utilisation using the 9-year-old cohort of the Growing Up in Ireland childhood longitudinal study

8,568 nine-year-olds and their primary carers were identified from a cluster random sample of 910 primary schools in the Republic of Ireland. The main outcome measures were: number of contacts with general practitioners (GPs), other medical doctors and mental health professionals (MHPs) over 12 months; visits to ED over 12 months; nights spent in hospital over the child's lifetime.

Children who reported being victimised by bullying had significantly more contacts with GPs $(Z=3.2 ; p=0.001)$, MHPs $(\mathrm{Z}=4.8 ; \mathrm{p}<0.001)$, but not with other medical doctors, visits to $\mathrm{ED}$ or nights spent in hospital. Ongoing chronic illness or disability was the most significant factor contributing to higher frequency of contact with the GP $(\mathrm{OR})=2.4,95 \% 95 \%(\mathrm{CI}) 2.1-2.7(\mathrm{p}<0.001)$, but being victimised by bullying increased the number of contacts in children after adjustment for ongoing chronic illness/disability. (Odds Ratio (OR) 1.14; 95\% CI 1.03-1.26; $\mathrm{p}=0.009$ ), parental age and household occupational class. Multivariable analyses provided some evidence that boys who were victimised by bullying were less likely to engage with GPs than girls (OR $0.85 ; 95 \%$ CI $0.77-0.93$; $\mathrm{p}=0.001$ ), but were more likely to contact MHPs (OR 1.57; 95\% CI $1.29-1.91 ; \mathrm{p}<0.001)$.

Being victimised by bullying as a child is associated with higher utilisation of primary care services, in particular GPs and MHPs, irrespective of whether they have an established chronic medical condition.

\section{ROAD TRAFFIC COLLISIONS IN IRELAND 2005-2013 PATTERNS, OUTCOMES AND IMPACTS}

\author{
E. McGovern ${ }^{1}$, H. Johnson ${ }^{1}$, A. O’Farrell ${ }^{1}$, D. McKeown ${ }^{1}$, \\ F. Donohue ${ }^{1}$
}

\section{${ }^{1}$ Health Intelligence Unit, Health and Wellbeing, H.S.E}

Road traffic collisions (RTCs) are the leading cause of injury related deaths in Ireland (1). The patterns and outcomes of RTCs in Ireland were analysed in order to evaluate previous and guide future road safety policy.

RTC-related admissions 2005-2013 to hospitals using Hospital inpatient enquiry (HIPE) data and Road Safety Authority (RSA) data 2005-2012 on road traffic collisions were examined. Patient outcomes and hospital outputs were examined using parametric and nonparametric testing and logistic regression. Interrupted time series analysis was conducted to examine the impact of major events on RTCs.

The average age at admission was 33 years, with more males than females admitted. RTC-related admissions involving all road users decreased by $37.4 \%$ except for pedal cyclists (increased by $26.3 \%$ over the period). RTC location mapping revealed that fatal RTCs were concentrated in urban centres and surrounding areas. Introduction of random breath testing (RBT) had a statistically significant sustained effect on RSA reported fatalities involving car occupants, 12 months post-introduction there were 25.3 fewer fatalities per quarter ( $\mathrm{p}<0.05,95 \% \mathrm{CI}-47.79,-2.75)$. At 3 months post-introduction, the RSA Crashed Lives campaign had a statistically significant effect on RSA reported fatalities involving all road users (15.7 fewer fatalities p $0.02,95 \%$ CI $-28.64,-2.74)$.

Measures impacting on driver behaviour (e.g., RBT) had the most persistent effect on RTCs. Road safety interventions should continue to target the young. The increase in pedal cycle RTCs and the impact of urban development and commuting on RTCs require a better understanding to guide future policy.

\section{ANTHROPOGENIC DETERMINANTS AND DISTRIBUTION OF VEROTOXIGENIC E. COLI (VTEC) INFECTION IN IRELAND: A GEO- STATISTICAL STUDY}

\author{
C. ÓhAiseadha ${ }^{1}$, J. O’Dwyer ${ }^{2}$, P. Hynds ${ }^{3}$, H. Johnson ${ }^{1}$, U. Fallon ${ }^{4}$ \\ ${ }^{1}$ Health Intelligence Unit, Health and Wellbeing, HSE; ${ }^{2}$ University \\ of Limerick; ${ }^{3}$ Dublin Institute of Technology (DIT); ${ }^{4}$ Dept of Public \\ Health, HSE Midlands, Tullamore
}

This ecological study explored associations between the nationwide distribution of verotoxigenic E. coli (VTEC) infection and the distribution of livestock, septic tanks and private wells. Approximately $14-15 \%$ of the Irish population uses an unregulated private well (CSO 2012). The incidence of VTEC infection in Ireland is among the highest in the EU, with groundwater contamination from diffuse agricultural sources suspected as a major transmission pathway.

We collated an entirely novel database, linking data from the Computerised Infectious Disease Reporting (CIDR) system, Census 
2011 and Census of Agriculture 2010. Health Atlas Ireland was used to geo-reference 989 primary VTEC cases notified during 2008-2013, including 521 serotype O157. These were linked via Census enumeration area to data for private wells per head, septic tank density, cattle density and sheep density $\left(\right.$ per $\mathrm{km}^{2}$ ), for bivariate and multivariate analysis.

The cumulative incidence of VTEC O157 infection in rural areas $(19 / 100,000)$ was three times the calculated urban rate $(6.3 / 100,000)$ $[\chi 2(1)=131.047, \mathrm{p}<0.001]$. Multivariate analyses indicate that private wells per head of population $(\mathrm{p}<0.001)$ and cattle density $(\mathrm{p}=0.007)$ are significantly associated with VTEC O157 infection. The existence of a significant relationship between VTEC infection and septic tanks was inconclusive.

This work provides further evidence of the long-suspected association between VTEC infection and private wells in Ireland. While previous studies have demonstrated diffuse groundwater contamination with indicator organisms from agricultural sources, this is the first Irish study (and among the first internationally) to positively demonstrate an association between human VTEC infection and cattle density.

\section{ACCURACY AND RELIABILITY OF SELF- REPORTING FOR OSTEOPOROSIS AMONG ADULTS AGED 50 AND OLDER LIVING IN IRELAND}

\author{
B. Cosgrove ${ }^{1}$, M. $\operatorname{Codd}^{1}$, A. Drummond ${ }^{1}$ \\ ${ }^{1}$ UCD School of Public Health, Physiotherapy \& Population Science, \\ Dublin
}

Osteoporosis, a common chronic condition, has a significant public health impact due to its association with fragility fractures. Self-reporting is frequently used in clinical practice and epidemiologic research to ascertain patients' disease status but few studies have examined its accuracy and reliability for osteoporosis. The objectives of this study were to calculate the prevalence of both self-reported and heel quantitative ultrasound (QUS)-determined osteoporosis among adults aged 50+ in Ireland; to assess the accuracy of self-reported osteoporosis; and to determine level of agreement between self-reported and heel QUS-determined disease.

This is an observational cross-sectional study using data from The Irish Longitudinal Study on Ageing (TILDA wave 1) - a nationally representative study of adults aged $50+$ residing in Ireland. The subjective measure derived from self-reports of doctordiagnosed osteoporosis $(n=8,163)$, and the objective measure from heel QUS results $(n=4,996)$. For the purposes of this study, heel QUS was taken as the 'gold standard' for osteoporosis diagnosis. Accuracy of self-reporting was measured in terms of sensitivity and specificity. Kappa statistics were used to determine level of agreement.

Prevalence of self-reported and objectively measured osteoporosis were 9.6 and $7.9 \%$ respectively. Sensitivity of self-report was low $(16.2 \%)$ while specificity was high $(95.8 \%)$. Level of agreement between self-report and heel QUS results was poor $($ kappa $=0.12)$

The findings of this study suggest that self-reports of doctordiagnosed osteoporosis lack accuracy and reliability. Clinicians should not rely on self-report as a proxy for diagnosis, and the importance of objective measurement in epidemiologic studies is underscored.

\section{DIFFERENCES IN ATTITUDES AND BEHAVIOUR OF MEDICAL STUDENTS TOWARDS SKIN CANCER PREVENTION}

\author{
S. Heneghan ${ }^{1}$, S. Cornyn ${ }^{1}$, C. Hayes ${ }^{1}$ \\ ${ }^{I}$ Trinity College Dublin
}

Melanoma is a form of skin cancer. Among its risk factors are UV exposure, age and skin type. Preventive measures include avoidance of risk factors and protective behaviours. This study aimed to investigate medical students' attitudes and behaviour around melanoma and to identify any differences.

Our study was carried out using an online, anonymous survey of 1st, 2nd and 3rd year medical students. This was accompanied by a letter to the participants and an information leaflet. The results were analysed using SPSS (version 21).

Response rate was $31.2 \%$ (163/522). 33/34 (97.1\%) third years reported they would attend a GP if they noticed a change in a mole compared to $67 / 88(76.1 \%)$ second years and $27 / 40(67.5 \%)$ 1st years $(\mathrm{p}=0.007)$. Those with fairer skin types $(1$ and 2$)$ were more likely than those with darker skin (type 3 and darker) to apply sunscreen everyday or on sunny days including cold days (39 vs. 18.6\%) $(\mathrm{p}=0.02)$, wear Factor $30-50(74.1$ vs. $51.2 \%)(\mathrm{p}=0.03)$ and to reapply sunscreen 'once an hour' when sunbathing (31.2 vs. $16.3 \%)$ $(\mathrm{p}=0.03)$. There were no differences in these preventive practices by year.

Greater clinical knowledge is an important factor influencing intended behaviour among medical students regarding early detection of melanoma. Although those with fairer skin were more likely to take preventive measures regarding sun exposure than those with darker skin colour, practice of preventive measures by medical students was suboptimal. It is expected that the results will be used to educate medical students about melanoma.

\section{PVL-SA: MANAGEMENT OF A 2 HOUSEHOLD EXTENDED FAMILY OUTBREAK}

\author{
R. Snounou ${ }^{1}$, C. McGeary ${ }^{1}$, R. Spiers ${ }^{1}$, P. O’Hare ${ }^{1}$, L. Geoghegan ${ }^{1}$ \\ ${ }^{1}$ Public Health Agency
}

Public Health investigation revealed that a new Panton-ValentineLeukocidin Staphylococcus Aureus (PVL-SA) case was a common contact of two previously unassociated cases-his partner and mother. His mother had recurrent PVL-SA infections linked to a previous outbreak. Construction of an extended family tree revealed that over 14 months there had been 4 cases and 1 colonisation across the two involved households. Both households had undergone previous decolonisation with known compliance issues.

A literature review established optimum evidence-based methods for community decolonisation of PVL-SA. A teleconference was held with the families' General Practitioners and microbiology to establish current infection status, emphasise importance of decolonisation and compliance, and coordinate simultaneous decolonisation. Compliance was encouraged by using multiple communication methods to reinforce the importance and methods of decolonisation and by problemsolving any potential obstacles with key family members.

Decolonisation was completed and post-decolonisation screening of high risk contacts is in progress. Non-availability of prescribed preparations led to a 3-day delay in decolonisation in one family, and 
a bout of gastrointestinal illness delayed post-decolonisation screening in the other.

The response to this outbreak highlighted the importance of considering pets, headwear and uniforms during decolonisation. Overlooking these may lead to prolonged outbreaks. The literature review supported the prioritisation of household decontamination due to sustained colonisation of fomites and direct fomite-human infection.

\section{MANDATORY PSEUDOMONAS SURVEILLANCE IN AUGMENTED CARE AND NEONATAL SETTINGS IN NORTHERN IRELAND-WHERE ARE WE 2 YEARS LATER?}

\author{
R. Spiers ${ }^{1}$, L. Patterson ${ }^{1}$, C. McLoughlin ${ }^{1}$, L. Geoghegan ${ }^{1}$ \\ ${ }^{1}$ Public Health Agency
}

Pseudomonas is an important cause of healthcare-associated infection, particularly in patients who are very ill or immunocompromised. Individuals may be colonised on the skin, nose and throat, usually without problems. Infections of the bloodstream are, however, particularly serious. Pseudomonas surveillance in clinical augmented care settings (including neonatal care) was introduced in Northern Ireland in January 2013 following a recommendation arising after outbreaks in neonatal units.

All microbiologically confirmed bacteraemias and isolates from sterile sites from non-neonatal augmented care were reported. In neonatal units both colonisations and confirmed infections were reported. Information collected includes patient demographics, specimen details, risk factor/outcome data and VNTR typing. Surveillance data between 28th January 2013 and 31st January 2015 were extracted and analysed.

There were 64 positive Pseudomonas isolates reported from nonneonatal augmented care settings. The majority of isolates reported were from haematology units $(54.7 \%)$ and adult ICU (includes cardiac ICU; $17.2 \%)$ and oncology units (14.1\%). Typing information Results for 44 isolates showed that all isolates were unique.

Overall, 40 colonisations (from 20 neonates) have been reported from all neonatal units in NI. 26/40 isolates were $P$. aeruginosa with 4 isolates containing $P$. putida, 7 P. fluorescens and $2 P$. stutzerii. Through typing, one cluster has been identified since the beginning of the programme.

This programme provides a unique insight into patterns of Pseudomonas in augmented care settings through mandatory reporting. It is reassuring to see there have been no infections in neonatal units despite colonisation, and that no isolates have been linked to water/ environmental samples to date.

\section{PREPAREDNESS FOR EBOLA VIRUS DISEASE AMONG GENERAL PRACTITIONERS IN THE NORTH WEST OF IRELAND}

\section{A. O’Connor ${ }^{1}$ \\ ${ }^{1}$ Sligo Specialist Training Programme in General Practice}

General Practitioners (GPs) are in the frontline of the public health response to novel infectious diseases like Ebola Virus Disease (EVD) but little is known about their knowledge of EVD and preparedness to manage a potential case. Personal Protective Equipment (PPE) packs along with information sheets had been distributed by the Health Service Executive to the majority of GPs in August 2014.

A postal survey was sent to all GPs in the North West of Ireland in November 2014 to explore awareness of EVD.

$73 / 141(52 \%)$ of questionnaires were returned. 50/73 (69\%) felt unprepared to deal with a case of possible EVD. 27/73 (37\%) correctly identified the three countries with ongoing transmission of EVD in the current outbreak. 56/73 (77\%) had PPE packs in their practice. Only $3 / 73(4 \%)$ had received training or instruction in using PPE. For all three, this was written or online training only. 26/73 (36\%) knew how to don PPE correctly but only 6/73 (8\%) knew how to remove it.

GPs are expected to be prepared for any emergency. This survey has shown that most GPs feel unprepared to deal with a case of EVD and that they do not know how to safely use PPE. In any potential future pandemic, GPs are likely to be at the forefront of the response. This survey suggests that GPs need practical training which would include PPE use to feel comfortable with evaluating the risks posed by infectious patients.

\section{A COMPARISON OF ANTENATAL AND POSTNATAL MOTHER'S KNOWLEDGE ABOUT NEWBORN BLOODSPOT SCREENING}

\author{
C. Fitzgerald ${ }^{1}$, E. Heery ${ }^{1}$, N. Conneally ${ }^{2}$, B. Linnane ${ }^{3}$, S. George ${ }^{1}$, \\ P. Fitzpatrick ${ }^{1}$
}

${ }^{1}$ UCD School of Public Health, Physiotherapy \& Population Science, Dublin; ${ }^{2}$ University College Dublin, School of Medicine, Dublin; ${ }^{3}$ Dept of Paediatrics, University Hospital Limerick

National and international newborn bloodspot screening (NBS) programmes recommend that parents should receive information about NBS prior to screening. Research suggests that information provided to parents is unplanned and informal, with most received in the postnatal period. The aim of this study was to evaluate antenatal and postnatal women's knowledge about and attitudes towards NBS.

This was a cross-sectional study of 693 antenatal ( $\geq 36$ weeks gestation) and 379 postnatal women (post NBS) recruited from three large maternity hospitals in Ireland. Women were asked to selfcomplete an anonymous questionnaire about NBS. Logistic regression was used to identify demographic and maternity factors associated with knowledge.

Significantly more postnatal than antenatal women agreed that they understood everything about NBS (53.6 vs. 33.0\%; OR 2.36; 95\% CI 1.80-3.09) and agreed that 'NBS tests for serious inherited diseases' (81.0 vs. 60.1\%; OR 2.88; 95\% CI 2.10-3.98). Significantly more postnatal than antenatal women correctly disagreed with the statement 'NBS tests for common diseases like asthma and diabetes' (64.9 vs. $29.5 \%$; OR 4.44 ; $95 \%$ CI $3.35-5.88$ ). Only $22.6 \%$ of all women that completed the questionnaire recalled receiving the NBS booklet. Multiparity $(\mathrm{p}<0.001)$, higher education status $(\mathrm{p}<0.05)$, native English speaker $(\mathrm{p}<0.05)$ and being a postnatal mother $(\mathrm{p}<0.001)$ were independent predictors of better NBS knowledge.

Antenatal women in the third trimester have limited knowledge and awareness about NBS. Postnatal mothers have increased knowledge. However, there is a desire in both groups for more information about NBS. 


\section{ADHERANCE TO THE PRIMARY IMMUNISATION SCHEDULE IN PRETERM VERSUS TERM INFANTS}

\author{
C. Power $^{1}$ \\ ${ }^{1}$ Dept of Neonatology, Cork University Maternity Hospital
}

Preterm infants are more susceptible to infection, including vaccine preventable diseases. A delay in immunisation may adversely affect their health 1. Vaccinating preterm infants according to their chronological age, not their corrected gestational age, is recommended. Despite this, previous studies have demonstrated delays in the administration of immunisations to preterm infant groups 2 .

This retrospective case-control study measures timing of $2,4,6$, 12 and 13 month immunisations in preterm infants ( $\leq 32$ weeks gestation), compared to term infants ( $\geq 37$ weeks gestation). Preterm infants born in Cork University Maternity Hospital from January 2012 onward were included and matched with the next born term infant.

The HSE South immunisation database was accessed to record timing of immunisations. Infants immunised within one month of the recommended date were considered to be immunised on time.

Data was collected on 95 preterm infants and 92 matched term infants.

Table 1 Adherence to immunisation schedule in preterm and term infants

\begin{tabular}{llllll}
\hline $\mathrm{N}$ & 2 month & 4 month & 6 month & 12 month & 13 month \\
\hline Preterm & 85 & 69 & 58 & 52 & 18 \\
Term & 84 & 67 & 51 & 44 & 25 \\
\hline
\end{tabular}

$94 \%$ of preterm infants had completed 2, 4 and 6 month vaccines compared to $90 \%$ of term infants. $56 \%$ of preterm infants had completed all immunisations to 13 months compared to $64 \%$ of term infants.

Timing of 2, 4 and 6 month immunizations are largely compliant with no significant difference in adherence the recommended schedule in preterm and term groups. Vaccine adherence could be improved in both groups for 12 and 13 month immunisations.

\section{EBOLA VIRUS DISEASE-RESOURCE IMPLICATIONS FOR DEPARTMENTS OF PUBLIC HEALTH}

\author{
R. Glynn ${ }^{1}$, M. Boland ${ }^{1}$, H. Murray ${ }^{1}$ \\ ${ }^{I}$ Dept of Public Health, HSE East, Dublin
}

The recent outbreak of Ebola virus disease (EVD) in West Africa has brought issues of emergency planning and preparedness for emerging viral threats, including Ebola, pandemic influenza and MERS-CoV into focus here in Ireland. This study evaluates the implications of this work for Departments of Public Health, in terms of emergency planning, business continuity and human resources, and stakeholder engagement.

All eight Directors of Public Health were contacted in March 2015 and requested to answer a short questionnaire regarding EVD-related workload within their Department since the declared Public Health Emergency of International Concern in August 2014. Preparedness, planning, human resources, the development of local plans and written advice, and involvement with external stakeholders were included. The extent of participation in regional or national groups was assessed and respondents were requested to provide suggestions for improvements in future work. Qualitative analysis with key respondents from each department was carried out.

Results will be used to assess preparedness, the evolving response, multisectoral involvement and communication, and will inform the emergency planning response within Departments of Public Health in the future. This work will attempt to quantify the time and effort devoted to preparedness activities for EVD since August 2014. It will also attempt to identify the extent to which this work and the experiences so gained can be utilised to advance an integrated approach to future public health emergency management at both local and national level.

\section{IMPROVING EFFICIENCY AND REDUCING COSTS-A STUDY INTO CURRENT USE OF WARD STOCK ROOMS AND HOW A STANDARDISED APPROACH CAN BENEFIT ALL}

\author{
C. Fenelon ${ }^{1}$, S. Barry ${ }^{2}$ \\ ${ }^{1}$ Dept of Gastroenterology, St. James's Hospital; ${ }^{2}$ Dept \\ of Orthopaedics, St. James's Hospital
}

Hospital ward stock rooms are hives of activity both day and night, used by all hospital staff, including doctors, nurses and other allied health professionals (AHPs). Ward stock room layouts commonly differ between wards. Standardised approaches to ward stock rooms may have existed in the past however this is no longer the case.

A 10 question survey conducted over two weeks to assess attitudes and work habits around ward stock rooms had 111 respondents comprising 58.6\% Non-Consultant Hospital Doctors, 36.0\% Nurses \& $5.4 \%$ AHPs. Of the 111 respondents, $53.2 \%$ used the stock room more than 10 times a day. $94.6 \%$ had issues with stock rooms while $82 \%$ had trouble finding stock with $53.2 \%$ affected daily (Table 1 ).

Time spent looking for stock reduces efficacy, lengthens the working day and delays care to subsequent patients. This also has cost and EWTD compliance implications. For example, interns delayed a conservative thirty minutes a day totals $2.5 \mathrm{~h}$ a week which has a cost implication of over $€ 150 \mathrm{k}$ per annum $[2.5 \mathrm{~h} \times € 15.69$ (intern hourly rate) $\times 52$ weeks $\times 50$ interns $=€ 101,335 * 1.5$ (overtime $=$ time and a half) $=€ 152,002$ per annum). Savings across other staff grades have not been quantified.

We recognise that stock requirements and room dimensions differ throughout different wards but suggest that a general standardised layout of stock rooms throughout would benefit all. We believe that this simple measure would have significant time and cost savings in our tertiary referral hospital.

\section{REDUCING COSTS-PROPOSED BENEFITS OF CHANGING FROM SCHEDULED REPLACEMENT OF PERIPHERAL VENOUS CANNULAE}

\author{
S. Barry ${ }^{1}$ \\ ${ }^{1}$ Dept of Orthopaedics, St. James's Hospital
}

Intravenous (IV) cannulation is a common medical technique allowing venous access usually for administration of medications, IV fluids, 
blood products, etc. Current guidelines for changing IV cannulae in this tertiary referral hospital state they should be removed at 72-96 h. However routine replacement increases healthcare costs and staff workload and requires patients to undergo repeated invasive procedures. A recent Cochrane review 'found no difference in catheterrelated bloodstream infection or phlebitis rates whether peripheral intravenous catheters are changed routinely every $72-96 \mathrm{~h}$ or when clinically indicated' (1).

This study looks at the benefits of only changing cannulae when clinically indicated. Each cannulation costs a minimum of $€ 3.03$ in equipment costs and €3.92-€7.85 in intern labour costs (@€15.69 intern hourly rate \& $15 \mathrm{~min} /$ cannulation). Elimination of one cannulation/day based on time rather than clinical need has the potential to save this facility between $€ 90 \mathrm{k}$ and $€ 116 \mathrm{k}$ annually, freeing interns up for other work, contributing to a reduced workload and improving compliance with EWTD.

Nursing staff would benefit by removing need to track time and date of placement; the cannula and its surround would be inspected as normal during IV medication administration

Implementation of this change would positively impact healthcare budgets (equipment and labour costs), reducing intern workload and overtime bill and improving EWTD compliance. This would also positively impact the patient experience, reducing the number of invasive, painful procedures and reduce IV medication delivery delays if there is a delay in resiting cannulae.

\section{INVESTIGATION AND MANAGEMENT OF A CLUSTER OF MULTI-RESISTANT PVL- PRODUCING STAPHYLOCOCCUS AUREUS INFECTIONS IN NORTHERN IRELAND}

\author{
L. Geoghegan ${ }^{1}$, C. O’Gorman ${ }^{2}$, C. McGeary ${ }^{1}$, L. Patterson ${ }^{1}$, I. King ${ }^{2}$ \\ ${ }^{1}$ Public Health Agency; ${ }^{2}$ South Eastern Health \& Social Care Trust
}

Staphylococcus aureus (SA), a Gram positive bacterium, commonly colonises skin and mucosal surfaces. SA may cause invasive infection by entering the body through broken skin, use of medical devices or during medical precedures. PVL (Panton-Valentine Leukocidin) toxin is produced by SA and is strongly epidemiologically associated with virulent and transmissable strains of SA.

In 2013 two life-threatening infections caused by PVL-producing SA were identified in Northern Ireland. Both SA isolates were meticillin sensitive but displayed several other antibiotic resistances. Both subsequently typed as the novel t417 strain. A SA incident was declared, a cluster response team established and a complex epidemiological and microbiological investigation commenced.

Over 16 months a total of 72 cases of t417 PVL-SA infection were identified and managed. A majority of cases were identified in primary and community care settings. 10 cases had no identifiable risk factors. 11 cases were healthcare workers. The cluster response included partnership management of all cases, review of PVL and SA laboratory diagnostic practices, review of SA epidemiology in NI, dedicated screening programme in augmented care settings and detailed risk assessments of care homes with t 417 PVL-SA cases.

Learning arising from this response includes challenges relating to case and cluster recognition, management of complex cases, standardisation of SA diagnostic practice and working with best practice guidelines. The central importance of human factors in delivering a successful cluster response is identified by all partners involved in this incident.

\section{LIMITATIONS OF CURRENT IRISH DATA ON DEVELOPMENTAL DYSPLASIA OF THE HIP}

\author{
R. McDermott ${ }^{1}$ \\ ${ }^{1}$ Department of Public Health, HSE Midlands
}

Developmental dysplasia of the hip (DDH) is a condition estimated to be present in $1-3 \%$ of newborn babies. Successful conservative treatment with harnessing depends on early detection. Delayed identification of DDH leads to surgical intervention often involving a series of operations and can lead to long-term sequelae.

DDH was identified as a priority by the Paediatric Clinical Programme and a group led by Public Health was convened to review the screening pathways for DDH in Ireland. An exploration of potential data sources on the DDH incidence and current screening outcomes was undertaken. Such data is required to inform decisions on screening provision, to quantify resources potentially released by improved screening and to provide a baseline for future monitoring and evaluation of the screening programme.

Potential data sources included the Hospital In-Patient Enquiry, National Integrated Medical Imaging System, EUROCAT and Personal Health Record systems. Maternity Units, Orthopaedic services and Community Medical Services were also contacted.

This scoping exercise revealed the lack of good national data on DDH. The presentation will discuss in detail the deficits identified and potential solutions to address them such as the opportunities afforded by the new HSE information systems currently in development: the National Immunisation and Child Information System, the National Maternity Clinical Information System and the National Integrated Medical Imaging System.

\section{DIAGNOSIS AND REPORTING OF ACUTE FLACCID PARALYSIS IN IRELAND: JANUARY 2009-DECEMBER 2014}

\author{
P. Flanagan ${ }^{1}$ \\ ${ }^{1}$ Health Protection Surveillance Centre, Dublin
}

Acute Flaccid Paralysis (AFP) surveillance is used in the surveillance for poliomyelitis and remains the gold standard strategy for monitoring the progress of polio eradication. Ireland has been polio free since 1984 when one case was reported (1). AFP surveillance is a sensitive tool for detecting a potential case of polio and is a marker of the ability of a health system to identify and respond to imported polio in a timely and effective manner and for documenting the absence of poliovirus circulation for polio-free certification.

All suspected cases of AFP are notified to the Irish Paediatric Surveillance Unit (IPSU) by the diagnosing clinician. These cases are then reported to the Health Protection Surveillance Centre (HPSC). This study retrospectively reviewed all suspected AFP cases reported in Ireland in children $<15$ years of age between January 2009 and December 2014 against the expected number of cases per year, quality and completeness of case patient investigation, reporting, and final diagnosis.

A total of 43 suspected cases were reported, of which 35 were confirmed AFP cases. Among these 35 cases, Guillain-BarrÃ $\subset$ Syndrome (GBS) was the most frequently reported syndrome $(48.6 \%$, $\mathrm{n}=17$ ). The average AFP incidence was 0.73 per 100000 population $<15$ years. 
This study highlights the likely under-reporting of AFP cases between 2009 and 2013 with improvements in reporting of cases evident for 2014. Improving surveillance and reporting of AFP among children $<15$ years old is important in order to document the absence of wild poliovirus importation and transmission in Ireland.

\section{ANALYSIS OF PHYSICAL ACTIVITY LEVELS IN CHILDREN ATTENDING THE W82GO! CLINIC AT THE CHILDREN'S UNIVERSITY HOSPITAL, TEMPLE STREET}

\author{
S. Doyle ${ }^{1}$, D. Cahill ${ }^{2}$, S. Murphy ${ }^{1}$ \\ ${ }^{1}$ The Children's University Hospital, Temple Street; ${ }^{2}$ University \\ College Dublin
}

Childhood obesity is a national (1) and an international problem. The causes of it are complex and multi-factorial and the pathophysiology is poorly understood. Many factors have been cited including reduced physical activity, changing food habits, changing lifestyle habits, the obesiogenic environment and genetic factors. The aim of this audit is to look at parental awareness of the amount of activity required for health, to assess the amount of activity the children are partaking in and to look at sedentary activities which the children take part in.

The audit was performed on data collected at the initial assessment of children referred to a hospital based obesity clinic. A standardised form is completed on each child attending the clinic and the figures have been taken from it and analysed.

The audit showed that of the 111 forms reviewed $56 \%$ were girls and $44 \%$ were boys. $95 \%$ were over 5 years old. $23 \%$ of the parents were aware that children require at least $1 \mathrm{~h}$ of physical activity per day. $61 \%$ of patients felt they were inactive in their free time. $63 \%$ of children attending the clinic do not get 60 min of activity. $49 \%$ of the children get to school by bus/car.

There is a lack of awareness among patients and their families attending this obesity service of the required amount of activity for health. They are leading sedentary lives.

\section{NATIONAL CLINICAL GUIDELINES FOR DIAGNOSIS, STAGING AND TREATMENT OF PATIENTS WITH CANCER}

\author{
D. Faherty ${ }^{1}$, E. O’Toole ${ }^{2}$, G. Killeen ${ }^{3}$, L. Murphy ${ }^{4}$, E. Nolan ${ }^{5}$, \\ N. O'Rourke ${ }^{6}$
}

\section{National Cancer Control Programme (NCCP), Dublin}

Evidence-based practice guidelines for the diagnosis, staging and treatment of patients with breast cancer, prostate cancer and gestational trophoblastic disease were developed by the National Cancer Control Programme (NCCP) as a result of the National Cancer Strategy 2006.

The guidelines were developed to provide recommendations to clinicians in the Irish setting. The methodology employed was based on the steps of evidence-based practice (1). The guideline development group comprised a multidisciplinary team including radiologists, pathologists, surgeons, medical oncologists, radiation oncologists, palliative care consultants, librarians, researchers, project managers, health economists and a methodologist.

Clinical questions were developed in PICO format focusing on areas of clinical practice where there was identifiable variation in practice or where there was new or emerging evidence. Literature searches were conducted to identify current International guidelines and primary literature using a systematic literature review protocol developed by HSE librarians. The literature was appraised for validity and applicability and recommendations were formulated by clinicians and assigned grades based on the level of evidence and clinical expertise. Draft guidelines were circulated for national and international review, prior to submission for Ministerial endorsement. An implementation plan based on the Behaviour Change Wheel (2) was developed which outlines the facilitators and barriers to successfully implementing the recommendations.

The effects of change will be measured through key performance indicators and other audit criteria. Guidelines on the diagnosis, staging and treatment of patients with lung, colorectal, pancreatic and oesophageal cancers are in progress.

\section{LEGIONNAIRE'S DISEASE IN IRELAND, 2005-2013: HOW COMPLETE IS REPORTING?}

\author{
T. Kelly ${ }^{1}$, J. O’Donnell ${ }^{1}$, D. O’Flanagan ${ }^{1}$ \\ ${ }^{1}$ Health Protection Surveillance Centre, Dublin
}

Legionnaires' disease is a severe and sometimes fatal respiratory disease caused by infection with gram negative Legionella bacteria. Found in freshwater and soil, these bacteria contaminate man-made water systems and spread by inhalation of contaminated aerosols or occasionally by aspiration of contaminated water.

To evaluate the effectiveness of the national computerised infectious disease reporting (CIDR) surveillance system to capture the incidence of Legionnaires' disease in Ireland, we analysed two data sources: CIDR and hospital in-patient activity (HIPE) data (from hospitalised patients following discharge) accessed via Health Intelligence Ireland. Data between 2005 and 2013 were analysed by age, gender, setting and serogroup using inter-censal population estimates.

Most (59.8\%) of the cases reported in Ireland between 2005 and 2013 were travel-related and $91.2 \%$ were associated with Legionella pneumophila, serogroup 1 . The mean Irish annual notification rate was 2.51 cases per million population, which is much lower than the European gold standard rate of 20 per million (1). This rate and the mean annual hospitalisation rate (2.30 hospital discharges per million population) compared favourably. Similar age profiles were found, with the highest age-specific incidence rate of notifications in the 75-79 year age group and of hospitalisations in the 70-74 year age group. The male:female ratio was 1.5:1 for Legionnaires' disease notifications and 1.46:1 for hospitalisations.

Our study shows that cases being reported to CIDR reflect hospital activity which is re-assuring, but the possibility of under-detection of Legionnaires' disease as a cause of community acquired pneumonia in Ireland needs to be further explored.

\section{EBOLA IN IRELAND? HEALTH PROTECTION ACROSS BORDERS}

\author{
R. Glynn ${ }^{1}$, M. Boland ${ }^{1}$
}

${ }^{I}$ Dept of Public Health, HSE East, Dublin, On Behalf Of The Port Health Subgroup Public Health Medicine Communicable Disease Group (HSE)

This paper depicts the multisectoral work involved in advancing and improving port health preparedness following the recent West Africa 
outbreak of Ebola virus disease (EVD) in 2014. We describe public health actions undertaken involving the five designated ports and three designated airports in Ireland. Communication with both the health and non-health sector was vital and many important links were made.

The Port Health subcommittee of the Public Health Medicine Communicable Disease group worked to optimise Medical Officer of Health functions and the HSE multidisciplinary port health group complemented this work with liaison with Environmental health, Emergency Planning and Ambulance services.

Meetings were held with harbour masters and airport managers, with airline operators and with different port stakeholders such as immigration, customs, cargo personnel, cleaning personnel. We prepared and distributed written updates on risks, management of presentation of a person suspected of EVD on a flight or ship, cleaning, advice for cargo personnel. Leaflets and posters giving advice and information were prepared by HPSC and distributed nationally to ports and airports for staff and passengers. Linkages were made with the Dept of Health and Transport. US customs and border screening at Dublin and Shannon airports required public health support as did obtaining Maritime Declarations of Health.

The EVD threat to Ireland, while low, required considerable preparedness of port health functions; during the response the public health interface with ports and airports has been strengthened and we hope it will foster further development in a spirit of co-operation across sectors and disciplines.

\section{VACCINE HESITANCY IN IRELAND IN 2014- RISK PERCEPTIONS}

\author{
S. Cotter $^{1}$ \\ ${ }^{1}$ Health Protection Surveillance Centre, Dublin
}

Childhood vaccination is a safe and effective means of protecting children against vaccine preventable diseases. However, a minority of parents are vaccine hesitant and do not support vaccination for their children.

The objectives of this study were to explore reasons for nonvaccination among Irish parents who decided not to vaccinate their children. Information was sought on factors influencing their decisions, attitudes to health, health seeking behaviours and how parents perceived the threat of infectious diseases to their children versus vaccines. This study involved face-to-face or telephone interviews of volunteer parents who were recruited either by invitations from health professionals who knew of the study or by posters placed in public venues. Interviews were taped and transcribed and analysed by identifying emerging themes.

Eleven parents participated, nine mothers and two fathers; the median age was 38 (range $28-48$ years); 9 were college or university educated; five were practitioners of complementary or alternative medicine (CAM); all parents reported using CAM for themselves and their children. Eight parents had never vaccinated any of their children, three had vaccinated some with selected vaccines. There was a belief that the human body could deal with infection and some reported a belief that children benefited from infection. The perception of risk associated with vaccine preventable diseases was quite low but the risk perception about vaccines was high

The results of this study will inform communications with parents who are exhibiting vaccine hesitancy and seek to address misinformation that circulates in the public domain.
PROSTATE CANCER: DOES MORE

INVESTIGATION AND TREATMENT RESULT IN BETTER HEALTH? A COMPARISON OF HEALTH OUTCOMES IN MEN WITH PROSTATE CANCER IN NORTHERN IRELAND AND THE REPUBLIC OF IRELAND

\section{A. Gavin ${ }^{1}$}

\section{${ }^{1} N$. Ireland Cancer Registry}

Prostate Cancer (PCa) levels are higher in Republic of Ireland (ROI) than N. Ireland (NI) (2011-44\%). Since 1994, 18,000 additional men have been diagnosed in ROI, 4,500 in NI). PCa mortality is similar. This allowed us to investigate different intensities of PCa diagnosis on men's health.

Postal questionnaire to $\mathrm{PCa}$ survivors up to 18 years post-treatment measuring asymptomatic vs. symptomatic), and seven ongoing physical adverse effects. Survivors were analysed separately for ROI and NI, by 'late disease' defined as stage III/IV and any Gleason Grade (GG) at diagnosis, and 'early disease' defined as stage I/II and GG 2-7.

3,348 (54\%) men responded. ROI responders were younger); more likely to present asymptomatically (66 vs. $41 \%$ ); without comorbidities (45 vs. 58\%), and with earlier disease Current Hormone Therapy $18 \%$ NI, 9\% ROI. Levels of incontinence (16 and impotence $(57 \%)$ were similar in early disease, only bowel problems $(\mathrm{NI}=21 \%, \mathrm{ROI}=12 \%)$ and fatigue $(\mathrm{NI}=29 \%, \mathrm{ROI}=17 \%)$ were significantly different. Multivariate modelling explained fatigue as treatment. In late disease NI vs. ROI men reported higher levels of libido loss ( 65 vs. $52 \%$ ) breast changes ( 23 vs. $9 \%$ ) hot flashes (41 vs. $19 \%$ ) and fatigue (39 vs. 25\%) which remained in multivariate modelling. However, these differences were not present when men on HT were analysed separately.

While men in NI had higher HT symptoms the increased intensity of testing has resulted in many additional men with incontinence and/ or impotence in ROI.

\section{PREVENTIVE HEALTH COUNSELLING DURING ANTENATAL CARE USING THE PREGNANCY RISK ASSESSMENT MONITORING SYSTEM (PRAMS) IN IRELAND}

\author{
M. Murphy ${ }^{1}$, L. O’Keeffe ${ }^{1}$, S. McHugh ${ }^{1}$, P. Corcoran ${ }^{1}$, R. Greene ${ }^{1}$, \\ P. Kearney ${ }^{1}$ \\ ${ }^{1}$ University College Cork
}

Antenatal care (ANC) provides an ideal opportunity to encourage behaviour change (1). Little is known about preventive health counselling provided during ANC. The Pregnancy Risk Assessment Monitoring System (PRAMS) in Ireland is a modified version of CDC methodology that monitors maternal behaviours and experiences that occur before, during and after pregnancy (2).

To assess the prevalence of preventive health counselling during ANC in pregnancy

Cross-sectional analysis of the PRAMS study was conducted at Cork University Maternity Hospital; a sampling frame of $\sim 2,400$ was used to sample 1,200 women. Outcome measures included selfreporting of preventive health counselling during ANC (smoking, alcohol, breastfeeding, appropriate weight gain and folic acid). 
Women were defined as in high need of counselling if they reported one of the following: cigarette/alcohol use, BMI $>25 \mathrm{~kg} / \mathrm{m}^{2}$, nonadherence to folic acid and intent to bottle-feed. Descriptive and bivariate analyses were conducted.

Response rate was $61 \%(\mathrm{n}=718)$; mean age was 32 years. Highest reported counselling rates were on breast-feeding $(84.8 \%$, $\mathrm{n}=592)$ and folic acid $(67.7 \%, \mathrm{n}=483)$; rates were lower regarding smoking $(47.6 \%, \mathrm{n}=333)$, alcohol $(48.5 \%, \mathrm{n}=338)$ and appropriate weight gain $(31.5 \%, \mathrm{n}=219)$. Women with a high need for counselling on breast-feeding $(30.7 \%, \mathrm{n}=219)$, alcohol consumption $(31.8 \%, \mathrm{n}=225)$ and appropriate weight gain $(29.2 \%, \mathrm{n}=202)$ were not significantly more likely to receive counselling. Women who took folic acid were almost twice as likely to have received advice $(\mathrm{OR}=1.9,95 \%$ CI $1.3-2.8, \mathrm{p}<0.05)$. The prevalence of preventive health counselling is lower than expected, improved delivery methods during pregnancy may be required.

\section{INFLUENZA SURVEILLANCE IN IRELAND DURING THE 2014/2015 SEASON; IMPACT OF INFLUENZA A(H3) VIRUSES ON MORBIDITY AND MORTALITY}

L. Domegan ${ }^{1}$, J. O’Donnell ${ }^{1}$, P. Flanagan ${ }^{1}$, D. O’Flanagan ${ }^{1}$,

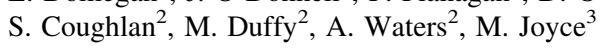

${ }^{1}$ Health Protection Surveillance Centre, Dublin; ${ }^{2}$ National Virus Reference Laboratory, UCD; ${ }^{3}$ Irish College of General Practitioners, Dublin

Influenza $\mathrm{A}(\mathrm{H} 3)$ viruses predominated in Ireland during the $2014 / 2015$ season. The majority of circulating $\mathrm{A}(\mathrm{H} 3)$ viruses characterised differed from the northern hemisphere vaccine. The objective was to determine the impact of circulating influenza viruses on morbidity and mortality.

A network of sentinel general practices report on all influenza-like illness (ILI) consultations. Other influenza surveillance indicators include: virology data; GP out-of hours calls; notifications; enhanced surveillance of hospitalised paediatric and critical care cases; hospital admissions; outbreak surveillance; mortality data and vaccine effectiveness studies.

ILI rates peaked during 72015 at 70.4 per 100,000 population, the highest peak rate since 2010/2011. The total number of confirmed influenza cases hospitalised this season is the highest reported since 2010/2011. Age-specific rates for confirmed influenza hospitalised cases were highest in those aged less than one year and those aged 65 years and older. For influenza admissions to critical care units, the highest age-specific rate was in those aged 65 years and older. The majority of influenza outbreaks affected the elderly in residential care facilities, with the highest number of outbreaks recorded since the 2009 pandemic. Excess mortality from all causes was reported in those aged 65 years and older. Interim influenza vaccine effectiveness estimates for Ireland were low.

This season was more severe than recent seasons, with increased ILI peak rates, hospitalisations, outbreaks and excess deaths, particularly in the elderly. The mismatch between the influenza $\mathrm{A}(\mathrm{H} 3)$ vaccine and circulating strain occurred globally and has highlighted the importance of early treatment with antivirals for risk groups. 